For Internal Distribution Only

\begin{abstract}
Accelerator Division
Alternating Gradient Synchrotron Department BROOKHAVEN NATIONAL LABORATORY

Upton, New York 11973
\end{abstract}

Accelerator Division

Technical Note

AGS/AD/Tech. Note No. 424

THE DIPOLE FIELDS OF THE AGS MAIN MAGNETS

R. Thern and E. Bleser

January 26, 1996 


\title{
THE DIPOLE FIELDS OF THE AGS MAIN MAGNETS
}

\author{
R. Thern and E. Bleser
}

\begin{abstract}
SUMMARY
This note records and parameterizes the strength of the dipole fields in the AGS main magnets.
\end{abstract}

\section{THE AGS MAIN MAGNETS}

The AGS main magnets are combined function $\mathrm{C}$-magnets which have two lengths. The "long" magnets are 90 inches long and are designated " $\mathrm{A}$ " or " $\mathrm{C}$ " magnets. The "short" magnets are 75 inches long and are designated " $\mathrm{B}$ " magnets. If the wide side of the magnet gap is to the outside of the magnet, away from the backleg, the magnet is called an open magnet. If the narrow side of the gap is to the outside of the magnet, the magnet is called a closed magnet. There are three kinds of magnets: long, open or closed, and short, open. Their designations are:

$$
\begin{array}{ll}
\text { A } & \text { long, open } \\
\text { B } & \text { short, open } \\
\text { C } & \text { long, closed. }
\end{array}
$$

Depending on their installation orientation in the ring, each of these magnets may be focussing or defocussing. Therefore there are six designations for magnets installed in the ring: $\mathrm{AD}, \mathrm{AF}, \mathrm{BD}$, $\mathrm{BF}, \mathrm{CD}$, and $\mathrm{CF}$.

\section{THE MEASUREMENT DATA}

Some years ago one of us (R.T.) made point to point field maps for an open magnet and a closed magnet over a number of current settings. The data were analyzed and used to generate the excitation functions and harmonic contents for the AGS main magnets. The results of this analysis have been incorporated into a number of operating systems, however the results have never been documented. Since magnetic storage may be long-lasting but the retrieval hardware and software seem to disappear in less than a decade, this note is being prepared to provide a permanent record of the data. It deals only with the analyzed results of dipole component of the main AGS magnets.

\section{THE RESULTS}

Tables $1 \mathrm{~A}, 1 \mathrm{~B}$, and $1 \mathrm{C}$ give the central dipole field strength for magnets $\mathrm{A}, \mathrm{B}$, and $\mathrm{C}$ for a number of different currents. The integration is performed along two different paths in each magnet: $\mathrm{F}$ for integration along the curved central orbit when the magnet is focussing, and $\mathrm{D}$ for integration along the curved central orbit when the magnet is defocussing. These paths are 
defined in Tech. Notes 215 and 217. Table 2 records the integrated field all the way around the AGS by summing over all 240 magnets. Table 2 was transmitted to the AGS power supply group and has been incorporated into the Main Magnet control program.

\section{ACCURACY}

The point to point accuracy of the measurements is about one part in 5000. Integrating over the fields probably gives relative accuracies better than this. However the overall calibration of the apparatus is probably about $0.5 \%$. This is the number of interest when Table 2 is compared against data that may be taken on the AGS with the Gauss clock. In this note we carry a lot of decimal places for two reasons: we can avoid round-off problems in the parameterizations and many decimal places will enable us to identify the source of the data in the future.

\section{PARAMETERIZATION}

Figure 1 shows the data from Table 2 plotted as $B \times L / I$ versus I. We have parameterized this data by fitting the low field data to powers of $1 / \mathrm{I}$, forcing the curve to be flat in the I range around 1000 Amperes, and fitting the remainder to a power series in I. The fitted curve is also shown in Figure 1 and the parameters are given in Table 3.

$$
\begin{gathered}
\frac{B \times L}{I}=\frac{a_{-4}}{I^{4}}+\frac{a_{-3}}{I^{3}}+\frac{a_{-2}}{I^{2}}+ \\
+a_{0}+a_{1} I+a_{2} I^{2}+a_{3} I^{3}+a_{4} I^{4}+a_{5} I^{5}+a_{6} I^{6}
\end{gathered}
$$

The inverse of these results, $I /(B \times L)$ versus $(B \times L)$, is given in Figure 2 and Table 4 . Since the momentum, $\mathrm{P}$, is directly proportional to $\mathrm{B} \times \mathrm{L}$, the parameterization for $\mathrm{P}$ is also given.

\section{BENDING RADIUS}

The bending radius of the AGS depends to some degree on how it is calculated. To get a somewhat general answer, we take each magnet, its effective length, and its bend angle from Tech Note 215 to find the arc length of the orbit in the magnet. Summing over the 240 magnets and dividing by 2 pi gives the bending radius:

\section{BENDING RADIUS $=85.380840$ merers}

This value should be consistent with other calculations to about 0.5 millimeters. 
TABLE 1A

\begin{tabular}{|c|c|c|c|c|}
\hline & \multicolumn{2}{|c|}{ MAGNET AD } & \multicolumn{2}{|c|}{ MAGNET AF } \\
\hline I & BO & $B O * L$ & BO & $B O * L$ \\
\hline Amperes & Gauss & Gauss*m & Gauss & Gauss*m \\
\hline 0 & 16.52 & 44.72 & 16.80 & 44.62 \\
\hline 30 & 80.29 & 198.44 & 81.82 & 198.45 \\
\hline 32 & 84.36 & 208.32 & 85.96 & 208.33 \\
\hline 34 & 88.64 & 218.64 & 90.33 & 218.66 \\
\hline 38 & 97.29 & 239.54 & 99.15 & 239.57 \\
\hline 60 & 145.04 & 354.72 & 147.84 & 354.84 \\
\hline 100 & 232.25 & 565.42 & 236.75 & 565.69 \\
\hline 108 & 249.78 & 607.66 & 254.63 & 607.97 \\
\hline 116 & 267.16 & 649.46 & 272.34 & 649.79 \\
\hline 360 & 807.11 & 1955.38 & 822.84 & 1956.58 \\
\hline 1000 & 2234.93 & 5413.36 & 2278.57 & 5416.81 \\
\hline 2650 & 5922.02 & 14337.76 & 6037.72 & 14347.22 \\
\hline 3550 & 7926.60 & 19176.77 & 8081.42 & 19190.55 \\
\hline 4450 & 9907.63 & 23916.61 & 10100.98 & 23937.56 \\
\hline 4800 & 10654.38 & 25681.18 & 10862.15 & 25706.30 \\
\hline 5150 & 11352.72 & 27317.92 & 11573.57 & 27347.74 \\
\hline 5500 & 11948.71 & 28706.41 & 12179.98 & 28740.30 \\
\hline 5850 & 12493.08 & 29963.29 & 12733.20 & 30000.93 \\
\hline
\end{tabular}


TABLE 1B

\begin{tabular}{|c|c|c|c|c|}
\hline & \multicolumn{2}{|c|}{ MAGNET BD } & \multicolumn{2}{|c|}{ MAGNET BF } \\
\hline I & BO & $B 0^{*} \mathrm{~L}$ & BO & $B O * L$ \\
\hline Amperes & Gauss & Gauss*m & Gauss & Gauss* m \\
\hline 0 & 16.75 & 38.49 & 16.97 & 38.42 \\
\hline 30 & 80.66 & 167.69 & 81.85 & 167.70 \\
\hline 32 & 84.73 & 176.00 & 85.98 & 176.01 \\
\hline 34 & 89.02 & 184.68 & 90.34 & 184.70 \\
\hline 38 & 97.70 & 202.25 & 99.14 & 202.28 \\
\hline 60 & 145.55 & 299.07 & 147.72 & 299.16 \\
\hline 100 & 232.94 & 476.24 & 236.44 & 476.42 \\
\hline 108 & 250.52 & 511.75 & 254.28 & 511.96 \\
\hline 116 & 267.91 & 546.85 & 271.94 & 547.08 \\
\hline 360 & 808.95 & 1645.12 & 821.16 & 1645.94 \\
\hline 1000 & 2239.78 & 4554.08 & 2273.65 & 4556.45 \\
\hline 2650 & 5934.98 & 12061.00 & 6024.77 & 12067.49 \\
\hline 3550 & 7944.10 & 16129.61 & 8064.25 & 16139.08 \\
\hline 4450 & 9929.28 & 20108.45 & 10079.33 & 20122.79 \\
\hline 4800 & 10677.76 & 21586.54 & 10839.00 & 21603.69 \\
\hline 5150 & 11377.74 & 22955.64 & 11549.14 & 22975.93 \\
\hline 5500 & 11974.52 & 24115.55 & 12153.99 & 24138.53 \\
\hline 5850 & 12520.35 & 25164.31 & 12706.70 & 25189.79 \\
\hline
\end{tabular}


TABLE 1C

\begin{tabular}{|c|c|c|c|c|}
\hline \multirow[b]{2}{*}{ I } & \multicolumn{2}{|c|}{ MAGNET CD } & \multicolumn{2}{|c|}{ MAGNET CF } \\
\hline & BO & $B O * L$ & BO & $B 0^{*} \mathrm{~L}$ \\
\hline Amperes & Gauss & Gauss*m & Gauss & Gauss*m \\
\hline 0 & 15.95 & 42.71 & 16.34 & 42.62 \\
\hline 30 & 79.48 & 196.24 & 81.11 & 196.29 \\
\hline 32 & 83.74 & 206.51 & 85.45 & 206.57 \\
\hline 34 & 88.74 & 217.69 & 90.55 & 217.76 \\
\hline 38 & 96.79 & 238.02 & 98.75 & 238.10 \\
\hline 60 & 145.64 & 355.95 & 148.55 & 356.15 \\
\hline 100 & 231.72 & 563.36 & 236.31 & 563.76 \\
\hline 108 & 249.13 & 605.31 & 254.07 & 605.75 \\
\hline 116 & 266.99 & 648.36 & 272.27 & 648.84 \\
\hline 360 & 805.84 & 1950.77 & 821.62 & 1952.43 \\
\hline 1000 & 2229.32 & 5395.74 & 2272.90 & 5400.36 \\
\hline 2650 & 5909.51 & 14291.15 & 6025.01 & 14304.24 \\
\hline 3550 & 7909.71 & 19110.15 & 8064.35 & 19129.03 \\
\hline 4450 & 9884.75 & 23829.66 & 10077.97 & 23857.10 \\
\hline 4800 & 10633.90 & 25595.50 & 10841.61 & 25627.80 \\
\hline 5150 & 11333.09 & 27233.20 & 11553.89 & 27269.90 \\
\hline 5500 & 11953.07 & 28671.57 & 12184.60 & 28712.92 \\
\hline 5850 & 12489.53 & 29904.21 & 12729.72 & 29950.46 \\
\hline
\end{tabular}


TABLE 2

\begin{tabular}{|r|r|r|r|}
\hline \multicolumn{1}{|c|}{ I } & B*L & B*L/I & P \\
\hline \multicolumn{1}{|c|}{ AMPERES } & TESLA METERS & $T^{*} \mathrm{M} / \mathrm{A}$ & GeV/c \\
\hline & & & 0.0474 \\
\hline 0 & 0.9932 & & 0.2122 \\
\hline 30 & 4.4465 & 0.148218 & 0.2229 \\
\hline 32 & 4.6724 & 0.146011 & 0.2344 \\
\hline 34 & 4.9127 & 0.144492 & 0.2566 \\
\hline 38 & 5.3770 & 0.141500 & 0.3814 \\
\hline 60 & 7.9925 & 0.133208 & 0.6058 \\
\hline 100 & 12.6976 & 0.126976 & 0.6510 \\
\hline 108 & 13.6444 & 0.126337 & 0.6964 \\
\hline 116 & 14.5956 & 0.125824 & 2.0956 \\
\hline 360 & 43.9212 & 0.122003 & 5.7993 \\
\hline 1000 & 121.5442 & 0.121544 & 15.3598 \\
\hline 2650 & 321.9186 & 0.121479 & 20.5416 \\
\hline 3550 & 430.5194 & 0.121273 & 25.6153 \\
\hline 4450 & 536.8564 & 0.120642 & 27.5075 \\
\hline 4800 & 576.5149 & 0.120107 & 29.2619 \\
\hline 5150 & 613.2840 & 0.119084 & 30.7722 \\
\hline 5500 & 644.9372 & 0.117261 & 32.1072 \\
\hline 5850 & 672.9162 & 0.115028 & \\
\hline & & & \\
\hline
\end{tabular}


TABLE 3

\begin{tabular}{|c|c|c|c|}
\hline & & $\begin{array}{c}B^{*} \mathrm{~L} / \mathrm{I} \\
\mathrm{T}^{*} \mathrm{M} / \mathrm{A} \\
\end{array}$ & $\begin{array}{c}\text { P/I } \\
\mathrm{GeV} / \mathrm{C} / / \mathrm{A}\end{array}$ \\
\hline FORMULA & TERM & COEFFICIENT & COEFFICIENT \\
\hline$a_{-4}$ & $1 / 1^{\wedge} 4$ & $3.833395 E+04$ & $1.829045 \mathrm{E}+03$ \\
\hline$a_{-3}$ & $1 / 1^{\wedge} 3$ & $-3.003303 E+03$ & $-1.432980 \mathrm{E}+02$ \\
\hline$a_{-2}$ & $1 / /^{\wedge} 2$ & $8.165383 E+01$ & $3.895986 \mathrm{E}+00$ \\
\hline$a_{-1}$ & $1 / 1$ & $-1.384148 \mathrm{E}-03$ & $-6.604247 \mathrm{E}-05$ \\
\hline$a_{0}$ & CONSTANT & $1.214860 \mathrm{E}-01$ & $5.796515 \mathrm{E}-03$ \\
\hline$a_{1}$ & 1 & $-4.545545 \mathrm{E}-07$ & $-2.168837 E-08$ \\
\hline$a_{2}$ & $I^{\wedge} 2$ & $9.586722 \mathrm{E}-10$ & $4.574156 \mathrm{E}-11$ \\
\hline$a_{3}$ & $1 \wedge 3$ & $-6.700589 \mathrm{E}-13$ & $-3.197083 E-14$ \\
\hline$a_{4}$ & $1 \wedge 4$ & $2.027363 \mathrm{E}-16$ & $9.673249 \mathrm{E}-18$ \\
\hline$a_{5}$ & $1 \wedge 5$ & $-2.658005 E-20$ & $-1.268226 \mathrm{E}-21$ \\
\hline$a_{6}$ & $1 \wedge 6$ & $1.052191 \mathrm{E}-24$ & $5.020365 E-26$ \\
\hline
\end{tabular}


TABLE 4

\begin{tabular}{|c|c|c|c|}
\hline \multicolumn{3}{|c|}{$\begin{array}{c}I /\left(B^{*} L\right) \\
A /\left(T^{*} M\right)\end{array}$} & $\begin{array}{c}\mathrm{I} / \mathrm{P} \\
\mathrm{A} /(\mathrm{GeV} / \mathrm{c})\end{array}$ \\
\hline TERM & COEFFICIENT & TERM & COEFFICIENT \\
\hline $1 /\left(B^{*} L\right)^{\wedge} 4$ & $-1.070694 E+03$ & $1 / P^{\wedge} 4$ & $-1.163023 E-01$ \\
\hline $1 /(B * L) \wedge 3$ & $5.836957 E+02$ & $1 / P^{\wedge} 3$ & $1.328827 \mathrm{E}+00$ \\
\hline $1 /\left(B^{*} L\right)^{\wedge} 2$ & $-1.119693 E+02$ & $1 / P^{\wedge} 2$ & $-5.342444 \mathrm{E}+00$ \\
\hline $1 /(B * L)$ & $1.282979 E+00$ & $1 / \mathrm{P}$ & $1.282979 \mathrm{E}+00$ \\
\hline CONSTANT & $8.224488 E+00$ & CONSTANT & $1.723725 E+02$ \\
\hline$B * L$ & $-3.408321 E-04$ & $\mathrm{P}$ & $-1.497127 \mathrm{E}-01$ \\
\hline$\left(B^{*} L\right)^{\wedge} 2$ & $7.313631 \mathrm{E}-06$ & $P^{\wedge} 2$ & $6.733027 E-02$ \\
\hline$\left(B^{*} L\right)^{\wedge} 3$ & $-5.642907 \mathrm{E}-08$ & $P^{\wedge} 3$ & $-1.088778 \mathrm{E}-02$ \\
\hline$\left(B^{*} L\right)^{\wedge} 4$ & $1.966953 \mathrm{E}-10$ & $\overline{P^{\wedge} 4}$ & $7.954074 \mathrm{E}-04$ \\
\hline$\left(B^{*} L\right)^{\wedge} 5$ & $-3.143464 \mathrm{E}-13$ & $P^{\wedge} 5$ & $-2.664177 \mathrm{E}-05$ \\
\hline$\left(B^{*} L\right)^{\wedge} 6$ & $1.897916 \mathrm{E}-16$ & $\overline{P^{\wedge} 6}$ & $3.371247 \mathrm{E}-07$ \\
\hline
\end{tabular}


Figure 1: $B^{*}$ L/I vs I

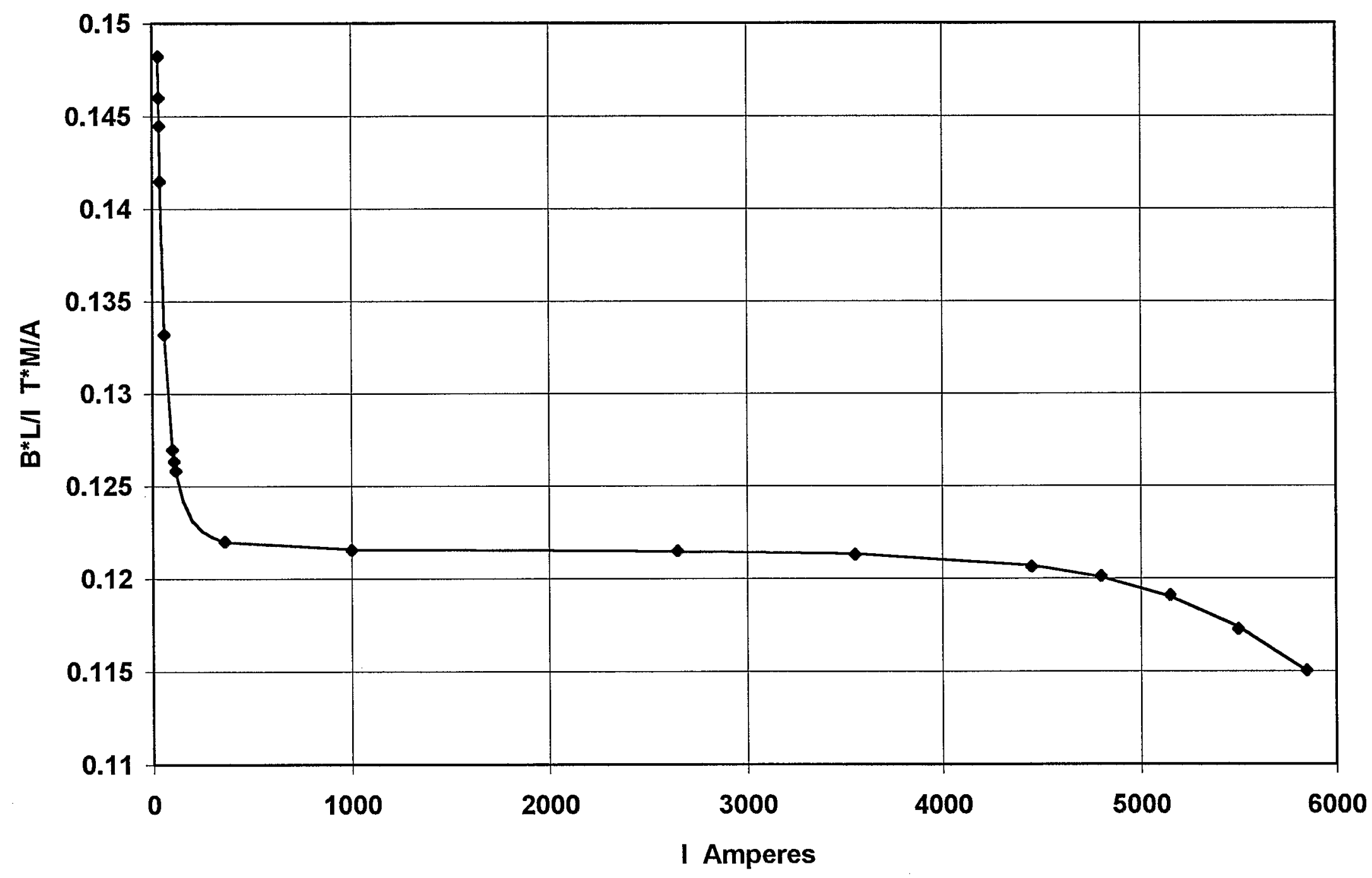


Figure 2: $I /\left(B^{*} L\right)$ vs $B^{*} L$

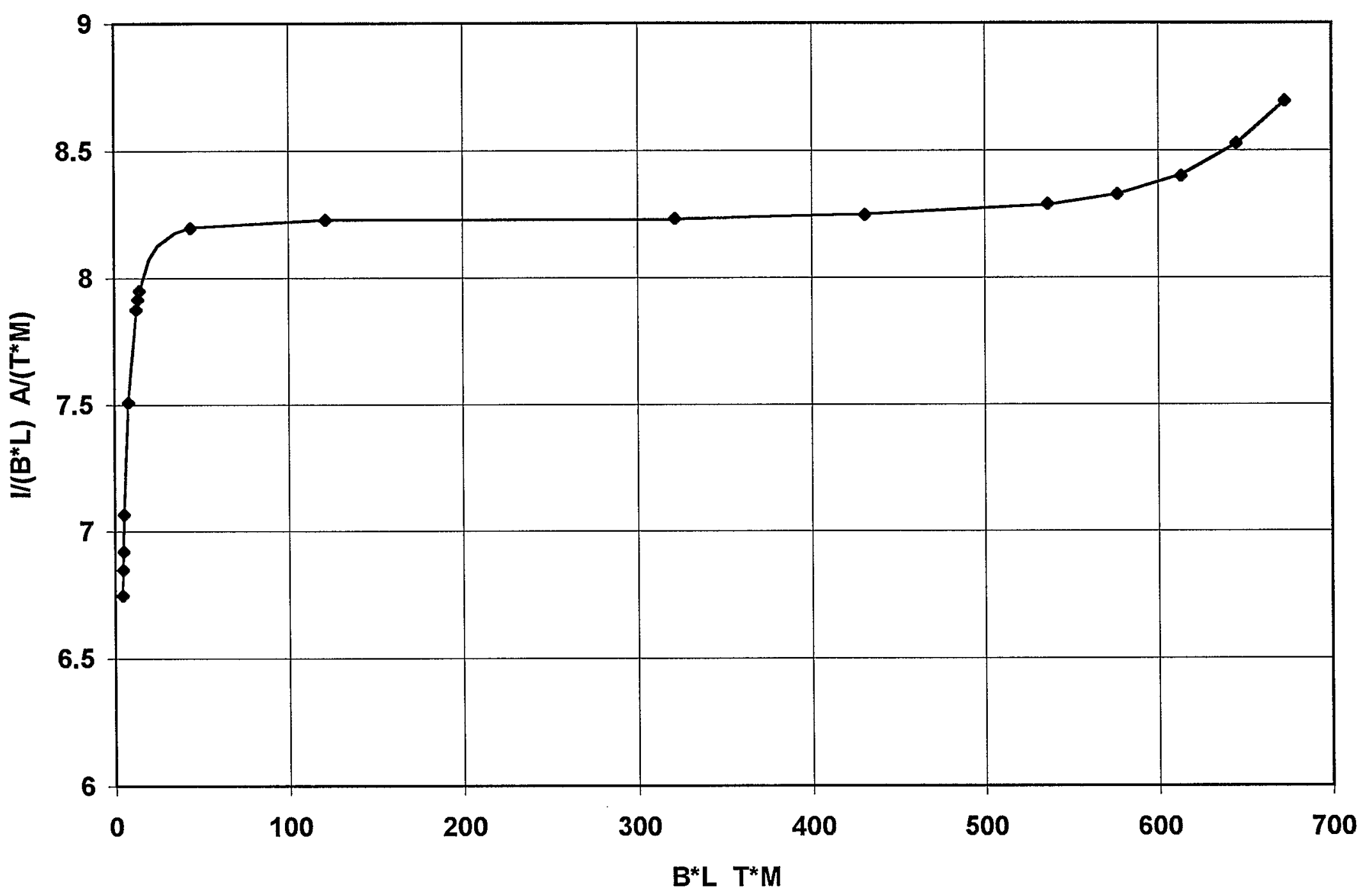

